\title{
4. AOTrauma Seminar West
}

Gereon Schiffer

\author{
„Was in Köln zweimal stattfindet, ist Tradition. Was dreimal stattfindet, ist Brauch- \\ tum.“ Geht es nach diesem Kölschen Grundgesetz, ist das AOTrauma Seminar West, \\ welches am 31. Januar 2018 stattfand, mittlerweile eingemeindet. Denn schon zum \\ vierten Male luden Dr. Gereon Schiffer (Bergisch Gladbach), Prof. Lars Müller (Köln) \\ und Prof. Joachim Windolf (Düsseldorf) in die Domstadt ein. Thema diesmal: \\ „Schaftfrakturen der oberen Extremität - von kompliziert bis komplikativ.“
}

Die Veranstalter verfolgten auch in diesem Jahr die Strategie, in einer „wochenendsparenden“ Mittwochsveranstaltung die 63 Teilnehmer einerseits durch 13 ausgewiesene Experten auf den neuesten Stand des Themas zu bringen und andererseits zum Nachdenken und Diskutieren auch über kontroverse Techniken und Verfahren anzuregen. Dank der hervorragenden Redner gelang dies problemlos.

Zunächst verschaffte Prof. Pol Rommens (Mainz) als weltweit anerkannter Experte der Oberarmmarknagelung einen Überblick über Historie und Weiterentwicklung dieses Implantats. Prof. Sascha Flohé (Solingen) ergänzte hinsichtlich "State of the art" der Plattenosteosynthese einschließlich der immer jungen Diskussion um den Radialisschaden, bevor Dr. Gereon Schiffer das Thema mit einer Betrachtung der konservativen Behandlungsmöglichkeiten komplettierte. Beginnend mit Böhlers Grundsatzrede „Gegen die operative Behandlung von Humerusschaftfrakturen“ von 1964 und über Sarmientos Ergebnisse führte die Diskussion zum heutigen Stellenwert der konservativen Therapie.

Nachdem hier bereits engagiert, aber immer kollegial und offen diskutiert wurde, stellte Dr. Christian Paul (Bonn) seine ersten Erfahrungen mit einem relativ neuen Implantat vor. Er hatte bereits in einigen ausgesuchten Fällen, vornehmlich bei palliativen Patienten, eine Oberarmstabilisierung mit lluminoss, einem durch Licht im Knochen aushärtenden Polymer durchgeführt. Auch wenn die Studienlage von Paul noch als äußerst dünn beschrieben wurde und eine deutliche Skepsis zu spüren war, kam das Plenum doch zu der Meinung, dass sich hier die Beobachtung der weiteren Entwicklung durchaus lohne.

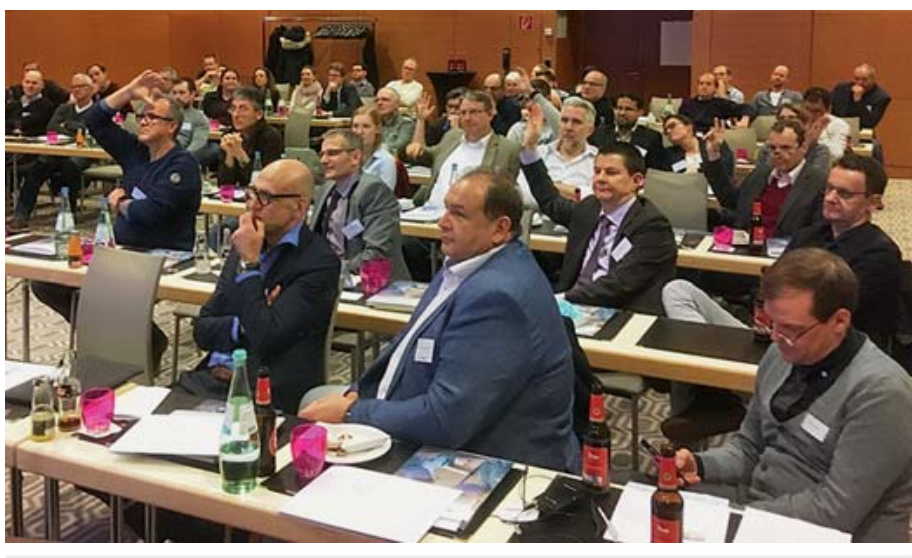

- Abb. 1 Teilnehmer im Hörsaal. (c) Gereon Schiffer

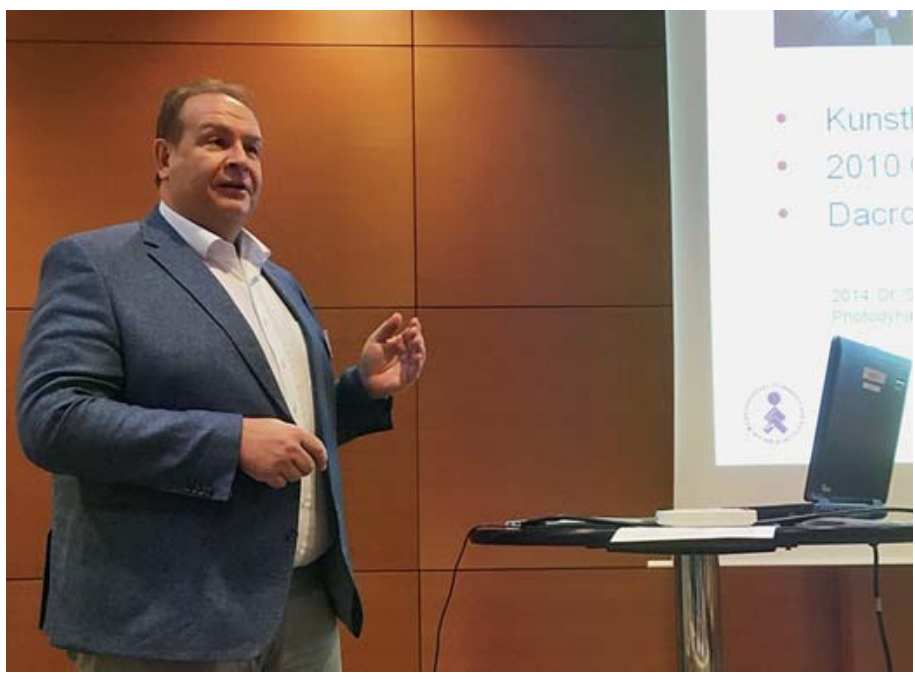

- Abb. 2 Christian Paul stellt Fälle vor. (c) Gereon Schiffer 
Die 2. Session drehte sich um die Schaftfrakturen des Unterarms. Dr. Christoph Faymonville, leitender Oberarzt der Uniklinik Köln, zeigte beeindruckende Bilder von komplizierten Frakturen und Weichteilproblematiken. Sein Schwerpunkt lag in der Beleuchtung der Plattenosteosynthese, die weiterhin als Goldstandard anzusehen ist. PD Dr. Axel Jubel (Köln, Eduardus KH) klärte anschließend die Frage, ob die intramedulläre Markraumnagelung (TEN) auch beim Erwachsenen angewendet werden könne. Sein Fazit: die Technik funktioniert exzellent bei Kindern und in vielen Fällen beim Adoleszenten. Beim Erwachsenen bleibt sie Einzelfällen vorbehalten. Prof. Tim Lögters (Köln, St. Antonius-KH) beschäftigte sich abschließend mit der Strategie und Therapie bei den nicht seltenen Unterarmschaft-Pseudarthrosen. Auch hier war wieder eine angeregte Diskussion zu verzeichnen.

Nach der Fingerfood-Pause, in der kaum jemand nach Hause ging, stellten Dr. Timm Schlummer (Warendorf) und Dr. Ulrich Thelen (Bergisch Gladbach) noch Fälle zur Diskussion. Schlummer setzte sich, ergänzend zum Vor- trag von Axel Jubel, mit der Geschichte und Realität der Unterarm-Verriegelungsnägel auseinander. Hier sind im Laufe der letzten Jahre viele Modelle vom Markt verschwunden, obwohl vorher durchaus gute Ergebnisse berichtet wurden.

Gemessen an den Evaluationsbögen verließen knapp 70 Unfallchirurginnen und Unfallchirurgen äußerst zufrieden den Saal. Es ist sicher davon auszugehen, dass die junge Brauchtumsveranstaltung auch in 2019 eine Fortsetzung finden wird.

Gereon Schiffer, Bergisch-Gladbach

Email: gereon.schiffer@vph-bensberg.de

Bibliografie

DOI https://doi.org/10.1055/a-0591-2676

OP-JOURNAL 2018; 34: 75-76 @ Georg Thieme Verlag KG Stuttgart · New York ISSN 0178-1715 